\title{
Localização de Usuários em Ambiente Interno Utilizando Abordagem de Sistema Multiagente
}

\author{
Ana Régia de M. Neves ${ }^{1}$, Humphrey C. Fonseca ${ }^{2}$, Letícia T. M. Zoby ${ }^{1}$, Célia G. \\ Ralha $^{2}$ \\ ${ }^{1}$ Faculdade de Tecnologia - Departamento de Engenharia Elétrica - Universidade de \\ Brasília (UnB) \\ Caixa Postal 4521 - 70904-970 - Brasília - DF - Brasil \\ ${ }^{2 \mathrm{II}}$ Instituto de Ciências Exatas - Departamento de Ciência da Computação - \\ Universidade de Brasília (UnB) \\ Caixa Postal 4466 - 70910-900 - Brasília - DF - Brasil \\ \{regianeves, letmaia\} @unb.br, humphrey.fonseca@caixa.gov.br, \\ ghedini@cic.unb.br
}

\begin{abstract}
This paper presents a simulation that provides indoor user location using the MultiAgent System approach. An architecture was extended and a prototype was developed on JADE platform with the definition and use of three contextual elements: identification, location and time. The focus of this work was user location, since it is considered a preliminary service to the development of context-sensitive applications, which should explore the context of different environments in order to provide more adaptive and userfocused services.
\end{abstract}

Resumo. Este artigo apresenta uma simulação para a oferta de serviço de localização de usuários em ambiente interno utilizando a abordagem de Sistema Multiagente. Neste trabalho, apresentamos a definição de uma nova arquitetura e o desenvolvimento de um protótipo com uso da plataforma $J A D E$, o qual utilizou três elementos contextuais primários: identificação, localização e tempo. O serviço de localização de usuários foi foco deste trabalho uma vez que é considerado preliminar para o desenvolvimento de aplicações sensiveis ao contexto, as quais devem explorar o contexto dos diversos ambientes com a finalidade de produzir serviços mais adaptativos $e$ voltados ao usuário.

\section{Introdução}

Diferente das aplicações tradicionais com características estáticas e baseadas em ambientes desktops, as aplicações sensíveis ao contexto exploram o contexto dos diversos ambientes para produzir serviços mais adaptativos e voltados ao usuário.

O objetivo é perceber e reagir às mudanças ocorridas no ambiente; sendo que o ambiente destas aplicações é dinâmico devido à mobilidade do usuário e caracterizado pelo uso intenso de dispositivos móveis multifuncionais, tais como smartphones.

Como os dispositivos e serviços deverão estar integrados no ambiente e cooperar entre si para desempenhar tarefas específicas, uma abordagem adequada a ser utilizada para desenvolver esse tipo de aplicação são os Sistemas Multiagentes (SMA). Um SMA 
é adequado para solucionar problemas difíceis de serem resolvidos por um agente individual ou um sistema monolítico [Faisal et al. 2010].

Este artigo apresenta uma nova arquitetura que tem como base a simulação de oferta de serviço de localização de usuários em ambiente interno [Neves et al. 2010]. O protótipo foi desenvolvido usando a plataforma JADE (Java Agent DEvelopment Framework), um software livre distribuído sob a licença LGPL, implementado em JAVA e baseado nos padrões FIPA (Foundation for Intelligent Physical Agents) [Bellifemine, Claire e Greenwood 2007].

O artigo está estruturado como segue: a Seção 2 apresenta os conceitos básicos sobre aplicações sensíveis ao contexto; a Seção 3 introduz a conceituação relacionada a área de SMA; a Seção 4 descreve alguns trabalhos correlatos; a Seção 5 apresenta a arquitetura e o protótipo desenvolvido; a Seção 6 apresenta o estudo de caso para o serviço de localização de usuário em um ambiente interno; e a Seção 7 encerra o artigo com as conclusões e trabalhos futuros.

\section{Aplicações Sensíveis ao Contexto}

Uma definição clássica de contexto é proposta em Dey e Abowd [1999] onde contexto é qualquer informação que caracteriza a situação de uma entidade, que pode ser pessoa, lugar ou objeto, considerada relevante em uma interação usuário-aplicação, incluindo o próprio usuário e a aplicação.

Segundo Coutaz et al. [2005], aplicações sensíveis ao contexto são capazes de monitorar e utilizar dinamicamente informações que provêm do ambiente ou do usuário. Neste sentido, Dey e Abowd [1999], Dey [2001] definem que contexto é utilizado para fornecer serviços e/ou informações aos usuários e a outras aplicações na realização de alguma tarefa.

Existem diversos elementos que podem ser utilizados para descrever um contexto, sendo que essa diversidade é dependente do domínio de cada aplicação. Por isso, alguns autores como Dey e Abowd [1999], Abowd e Mynatt [2000], Truong, Abowd e Brotherton [2001] propuseram a utilização de seis dimensões semânticas conhecidas como $5 \mathrm{Ws}+1 \mathrm{H}$ para auxiliar na especificação desses elementos, que são: (i) who? (quem?), identificar o usuário que está utilizando a aplicação; (ii) where? (onde?), localizar o usuário; (iii) when? (quando?), o momento que a interação está ocorrendo; (iv) what? (o quê?), a atividade corrente do usuário; (v) why? (por quê?), entender as razões das ações do usuário; e (vi) how? (como?), de que maneira as informações contextuais serão capturadas.

Dey e Abowd [1999] definem também a classificação dos elementos contextuais em primários e secundários; onde localização, identificação, atividade e tempo caracterizam o contexto primário, pois determinam a situação corrente de uma entidade particular. A partir disso, é possível responder quatro dimensões semânticas (quem, onde, quando e o quê) e derivar o contexto secundário. Por exemplo, com a localização do usuário determina-se quais as outras pessoas e/ou dispositivos estão próximos, além, de quais atividades estão ocorrendo naquela localização. Assim, os elementos contextuais enriquecem a solicitação explícita do usuário e, com isso, podem executar serviços mais próximos às suas necessidades [Vieira, Tedesco e Salgado 2009].

Conforme exposto, observa-se que o ambiente das aplicações sensíveis ao contexto devem ter como forte característica a dinamicidade, uma vez que o usuário 
movimenta-se de um local para outro, variando então as condições do ambiente com o decorrer do tempo, por exemplo: (i) condições físicas; (ii) recursos físicos e computacionais disponíveis; (iii) fontes de contexto; (iv) conectividade; e (v) movimentação de outros usuários e/ou recursos para dentro e para fora da área de interesse da aplicação [Loureiro et al. 2009].

Segundo Greenberg [2001], existem algumas dificuldades para o desenvolvimento dessas aplicações, a saber: (i) enumerar o conjunto de situações que podem existir em uma aplicação; (ii) identificar quais elementos contextuais podem determinar com precisão uma situação desse conjunto; e (iii) definir quais ações devem ser executadas em uma situação particular. Estas dificuldades podem ser bem tratadas com abordagens adaptáveis, dinâmicas e racionais, como as envolvidas em aplicações com agentes inteligentes.

\section{Abordagem de Sistema Multiagente}

Segundo Wooldridge [2002], para um agente ser considerado inteligente, ele deve ser autônomo, apresentar capacidade de comunicação, de cooperação, de raciocínio, deve ter mecanismos de planejamento para executar suas ações e ser adaptável quanto a mudanças ocorridas no ambiente. Também, deve ser capaz de prover serviços e alcançar objetivos individuais. Desta forma, Jih, Huang e Hsu [2009], consideram que esses atributos são adequados para o desenvolvimento de aplicações sensíveis ao contexto.

Segundo a definição de Vladoiu e Constantinescu [2010], um SMA é composto de múltiplos agentes inteligentes que interagem entre si. O uso deste paradigma também é indicado quando o domínio do problema é complexo, distribuído, imprevisível ou com diferentes objetivos [Sycara 1998; Stone e Veloso 2000]. Desta forma, o problema pode ser modulado permitindo que cada agente use o paradigma mais adequado para resolvêlo, além da interação e cooperação entre os agentes, caso o problema seja interdependente [Sycara 1998].

Stone e Veloso [2000] citam algumas das principais vantagens de se utilizar SMA: (i) paralelismo, é obtido através da atribuição de diferentes tarefas ou habilidades para diversos agentes no sistema; (ii) robustez, o sistema pode tolerar falhas de um ou mais agentes caso o controle e as responsabilidades estejam compartilhadas entre eles; e (iii) escalabilidade, o sistema é inerentemente modular facilitando a adição de novos agentes.

Segundo Weyns et al. [2007] um ambiente neste paradigma é definido como uma abstração de primeira classe que fornece as condições para os agentes existirem, além de mediar a interação entre eles e o acesso aos recursos. Por isso, definir suas propriedades é uma decisão de projeto que depende dos requisitos do domínio do problema. Os agentes precisam agir e perceber esse ambiente. Essa percepção pode ser limitada pela capacidade do próprio agente e pelas propriedades do ambiente.

\section{Trabalhos Correlatos}

Em Nguyen et al. [2009] foi desenvolvida uma aplicação, PlaceAware, que procura pessoas conhecidas ou amigos de amigos em lugares reais definido como comunidade social virtual (PBVC - Place-based Virtual Community) [Nguyen et al. 2008]. Essa aplicação utiliza a tecnologia Bluetooth para detectar a presença do usuário, onde cada dispositivo é identificado pelo endereço MAC e um nome amigável. A arquitetura desta 
aplicação pode ser dividida em três camadas, a saber: (i) a primeira camada é responsável por coletar as informações de contexto, foram definidos quatros agentes que detectam a presença do usuário ou dispositivo; (ii) a segunda camada é responsável pelo gerenciamento da comunidade virtual (PBVC), além de armazenar dados para uso posterior; e (iii) a terceira camada provê os serviços para a aplicação. Essa aplicação é baseada em sistema multiagente e desenvolvida na plataforma JADE. No momento, a nossa proposta arquitetural não está definida em níveis; no entanto, armazena informações do ambiente que estão sendo utilizadas na nova arquitetura proposta para localização do usuário.

Faisal et al. [2010] apresentam um sistema para segurança e controle ao acesso de prédios em uma universidade baseado em SMA e sensores sem fio. A arquitetura proposta é composta por duas categorias de agentes: (i) os agentes do sistema, que gerenciam a plataforma; e (ii) os agentes ACCESS [Robin et al. 2005], que são responsáveis pelos serviços sensíveis ao contexto. Em relação aos agentes do sistema existem dois agentes distintos, a saber: (i) o agente de diretório, que fornece o serviço de páginas amarelas para os agentes; e (ii) o agente gerenciador, que gerencia a criação e a exclusão dos agentes. Em relação à arquitetura ACCESS três agentes distintos foram adicionados, a saber: (i) o agente gerenciador de alarme recebe um sinal da rede sem fio e determina o código do sensor ativado, o código do prédio a partir da tabela sensor e envia para o (ii) agente de posição do prédio que consulta o nome e a posição do prédio a partir da tabela de prédios e envia essas informações para o (iii) agente de segurança que irá encontrar a menor distância entre o prédio que teve o alarme acionado e o guarda de segurança a partir da tabela de posição dos guardas de segurança. A nossa proposta não tem foco em segurança, no entanto, utiliza o serviço primário de localização e armazena informações de posição dos usuários para monitoramento em ambiente interno.

Em Neves et al. [2010] foi desenvolvido um protótipo baseado na abordagem SMA para estimar a localização de usuários em ambientes internos. Foram definidos três agentes reativos distintos: (i) agente cliente, que solicita a sua posição no ambiente; (ii) agente mapeamento, que procura quais agentes sensores estão disponíveis no ambiente para encontrar a localização do usuário; e (iii) agente sensor, que procura e encontra o usuário no ambiente. Para o desenvolvimento do protótipo foi utilizado a plataforma JADE. A arquitetura e o protótipo serviram como prova de conceito para a continuidade do desenvolvimento de um framework para localização e oferta de serviços em ambientes sensíveis ao contexto.

\section{Proposta de Oferta de Serviço de Localização de Usuários}

Com base na proposta apresentada em Neves et al. [2010] foi definida uma nova arquitetura para simulação de oferta de serviço de localização de usuários. Os elementos contextuais foram definidos baseados em três dimensões semânticas apresentadas em Dey e Abowd [1999], Abowd e Mynatt [2000], a saber: a identificação, a localização e o tempo. De acordo com esses elementos é possível controlar a identificação de todas as entidades participantes de uma atividade, no intuito de atender às necessidades dos usuários. Também com a combinação das dimensões de localização e tempo pode-se explorar não apenas a mobilidade dos usuários, mas também informações sobre a sua orientação em um ambiente físico e, consequentemente, fornecer serviços e/ou informações adaptados ao comportamento do usuário [Bulcão Neto 2006]. Além disso, 
esses elementos são armazenados para busca dos usuários no ambiente e futura especificação de grupos com permissões de acesso.

Para as simulações foram definidos três agentes distintos com a finalidade de localização em ambiente interno: usuário, ambiente e localizador. Os agentes usuários e localizador são reativos, onde suas ações são baseadas em regras pré-definidas; e o agente ambiente é reativo com registro de estados, onde este agente apresenta uma estrutura interna de registro de estados sobre os dados do ambiente que é utilizado para o processo de tomada de decisão [Russel e Norvig 2009]. A Tabela 1 exibe as definições de percepção e ações para cada agente definido, considerando que o ambiente nesta experimentação é acessível, estático, determinístico, não-episódico e discreto.

Tabela 1. Descrição das características de percepção e ação dos agentes

\begin{tabular}{|c|c|c|}
\hline Agente & Percepção & Ação \\
\hline $\begin{array}{l}\text { Usuário } \\
\text { (reativo } \\
\text { simples) }\end{array}$ & $\begin{array}{l}\text { - Receber informação de } \\
\text { localização proveniente do } \\
\text { agente ambiente } \\
\text { - Receber a informação de } \\
\text { localização de outro } \\
\text { usuário proveniente do } \\
\text { agente ambiente }\end{array}$ & $\begin{array}{l}\text { - Solicitar a própria localização para } \\
\text { o agente ambiente } \\
\text { - Solicitar a localização de outro } \\
\text { usuário para o agente ambiente }\end{array}$ \\
\hline $\begin{array}{l}\text { Ambiente } \\
\text { (reativo } \\
\text { com } \\
\text { registro de } \\
\text { estados) }\end{array}$ & $\begin{array}{l}\text { - Receber solicitação do } \\
\text { agente usuário } \\
\text { - Receber a localização do } \\
\text { usuário proveniente do } \\
\text { agente localizador }\end{array}$ & $\begin{array}{l}\text { - Identificar o usuário } \\
\text { - Solicitar a localização do usuário } \\
\text { para o agente localizador } \\
\text { - Armazenar posição do usuário no } \\
\text { log } \\
\text { - Informar ao agente usuário a } \\
\text { identificação e a localização } \\
\text { - Informar ao agente usuário a } \\
\text { localização de outro usuário }\end{array}$ \\
\hline $\begin{array}{c}\text { Localizador } \\
\text { (reativo } \\
\text { simples) }\end{array}$ & $\begin{array}{l}\text { - Receber a solicitação do } \\
\text { agente ambiente }\end{array}$ & $\begin{array}{l}\text { - Localizar o usuário } \\
\text { - Informar ao agente ambiente a } \\
\text { localização requisitada }\end{array}$ \\
\hline
\end{tabular}

O ambiente simulado é o prédio SG 11 do Departamento de Engenharia Elétrica da Universidade de Brasília que contém vários laboratórios. Para simulação, o ambiente foi reduzido a três salas (Sala 1, Sala 2 e Sala 3), nas quais os usuários podem se 
movimentar livremente em intervalos de tempo aleatório. A Figura 1 ilustra o ambiente utilizado na simulação.

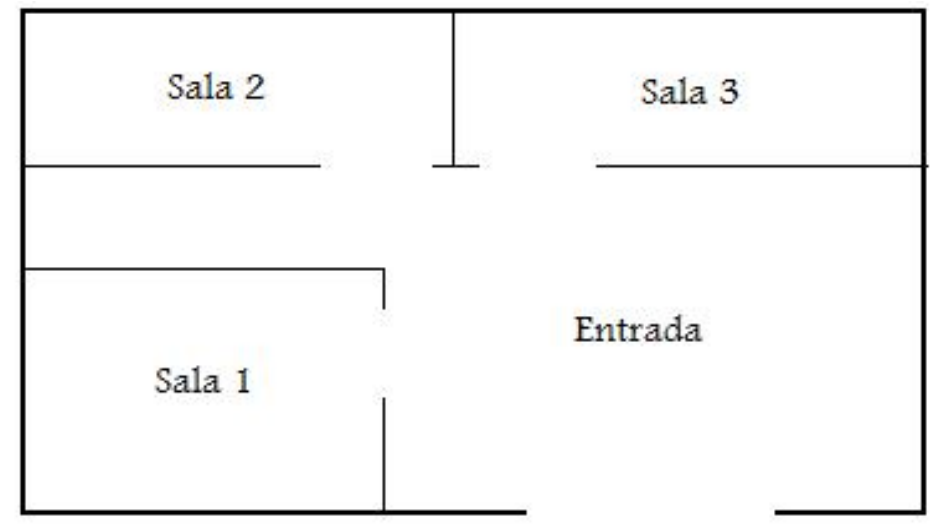

Figura 1. Ambiente do prédio SG 11 utilizado na simulação

\subsection{Arquitetura e Protótipo}

A Figura 2 apresenta a nova arquitetura, onde o agente usuário está dividido em três categorias: (i) agente usuário $p$ - composto por docentes e podendo variar de 1 a $n$ docentes presentes no prédio; (ii) agente usuário $a$ - composto por discentes e podendo variar de 1 a $x$ discentes; e (iii) agente usuário $f$ - composto por funcionários e podendo variar de 1 a $z$ funcionários presentes.

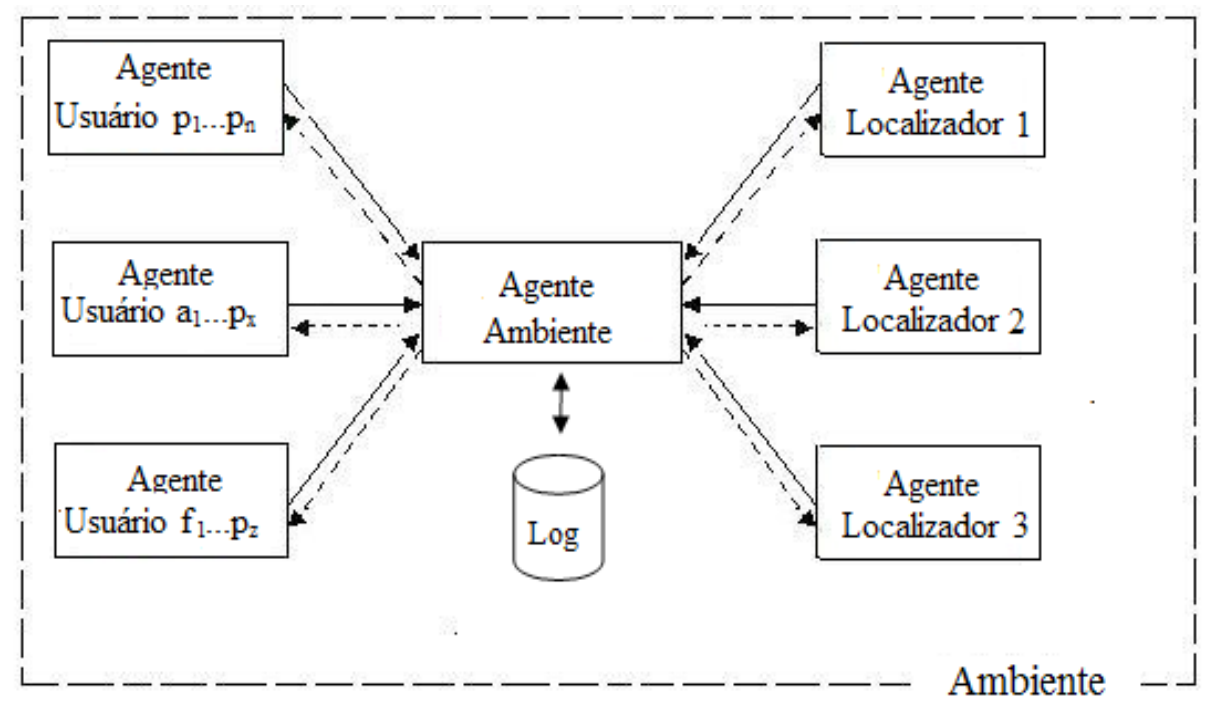

Figura 2. Arquitetura utilizada para simulação

Os agentes usuários podem solicitar a sua própria localização ou a de outro agente usuário ao agente ambiente e vão aguardar a resposta. $\mathrm{O}$ agente ambiente serve como interlocutor entre os agentes usuários e os agentes localizadores e inicia a busca de quais agentes localizadores estão disponíveis para localizar os agentes usuários. Cada sala do ambiente contém um agente localizador. Os agentes localizadores procuram e localizam os usuários no prédio SG 11, e então, repassam a resposta ao agente ambiente, 
o qual informa aos agentes usuários as localizações solicitadas.

A Figura 3 apresenta o cenário que envolve a interação dos agentes no ambiente, o "ID" é o número de identificação do usuário, "dt" é a data corrente, "h" é a hora que o sistema localizou o usuário, "sl" é a sala que o usuário se encontra, " $\rightarrow$ " representa a solicitação e "-->” representa a resposta dos agentes.

Observe que a sequência da interação ilustrada na Figura 3 é: (1) o agente usuário solicita sua localização ao entrar no ambiente; (2) o agente ambiente recebe a solicitação do agente usuário, o identifica com um ID de matrícula, procura quais agentes localizadores estão disponíveis com o serviço de localização e repassa a solicitação do agente usuário com o ID de matrícula aos agentes localizadores; (3) os agentes localizadores recebem a mensagem do agente ambiente com o ID do usuário, procuram o usuário no prédio e informam a localização solicitada ao agente ambiente; (4) o agente ambiente guarda a informação mais recente de localização no log, registrando a data, o horário e a sala onde o usuário se encontra; e (5) o agente ambiente envia a localização solicitada ao agente usuário.

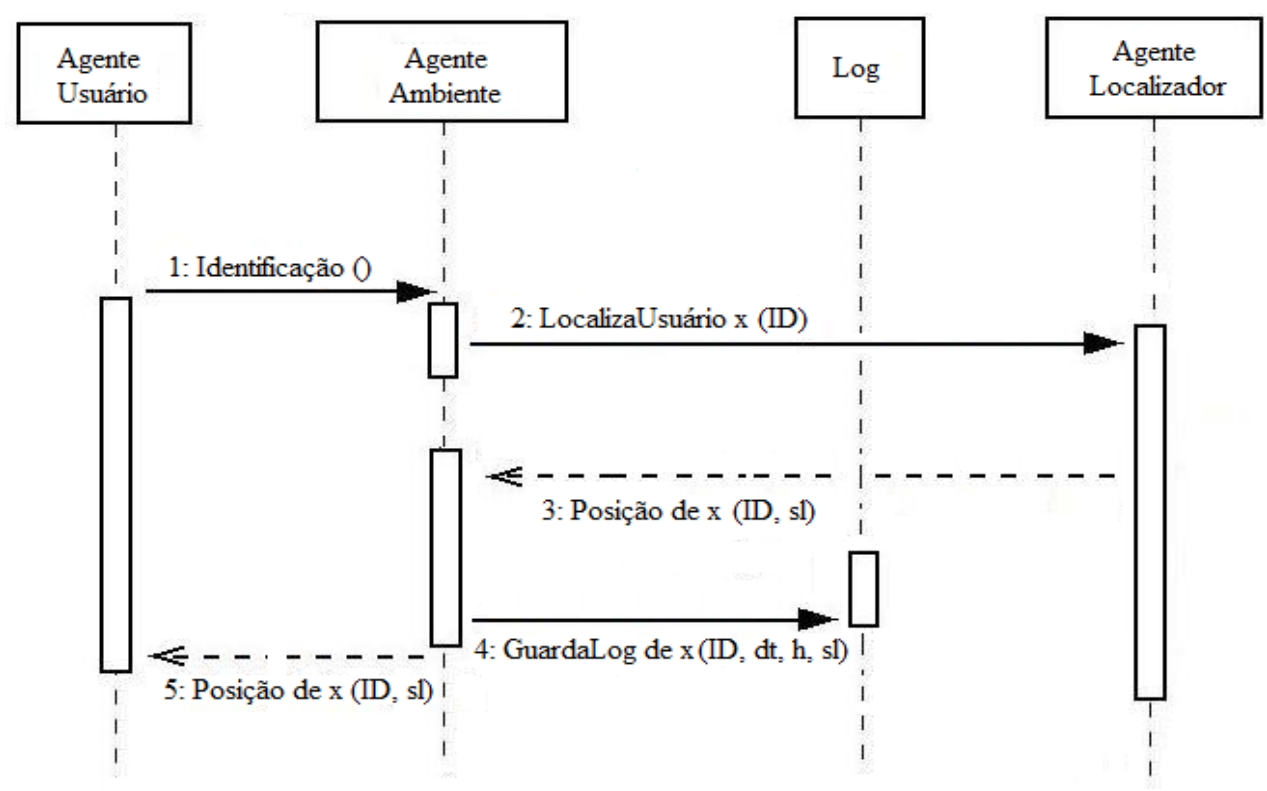

Figura 3. Diagrama de sequência

\section{Simulação de Localização}

Para validar a arquitetura proposta e o protótipo desenvolvido este trabalho foi realizada uma simulação para oferta do serviço de localização de usuário no prédio SG 11. Sendo que essa simulação ainda não inclui redes de sensores sem fio.

Imagine que um usuário solicite a localização de outro usuário no prédio, então o agente ambiente deve consultar o log onde estão armazenadas as localizações mais recentes dos usuários no prédio e informar ao usuário solicitante a informação de localização. Se ele não for encontrado, uma mensagem é enviada ao usuário solicitante informando que aquela pessoa não se encontra no prédio. Apenas usuários cadastrados poderão solicitar a localização de outro usuário. 
A Figura 4 apresenta um screenshot do console da usuária Régia que foi identificada com a $I d$ número 2 a fim de ser monitorada no ambiente. Sua primeira localização é na Sala 3. Em um certo momento, ela precisa encontrar a usuária Letícia para trocarem informações sobre o artigo que estão escrevendo e solicita ao agente localizador que a encontre. $\mathrm{O}$ agente localizador após verificar no $\log$, informa que a usuária Letícia foi identificada com a Id número 1 e está localizada na Sala 2. A partir desse momento, a movimentação da usuária Letícia será repassada para a usuária Régia.

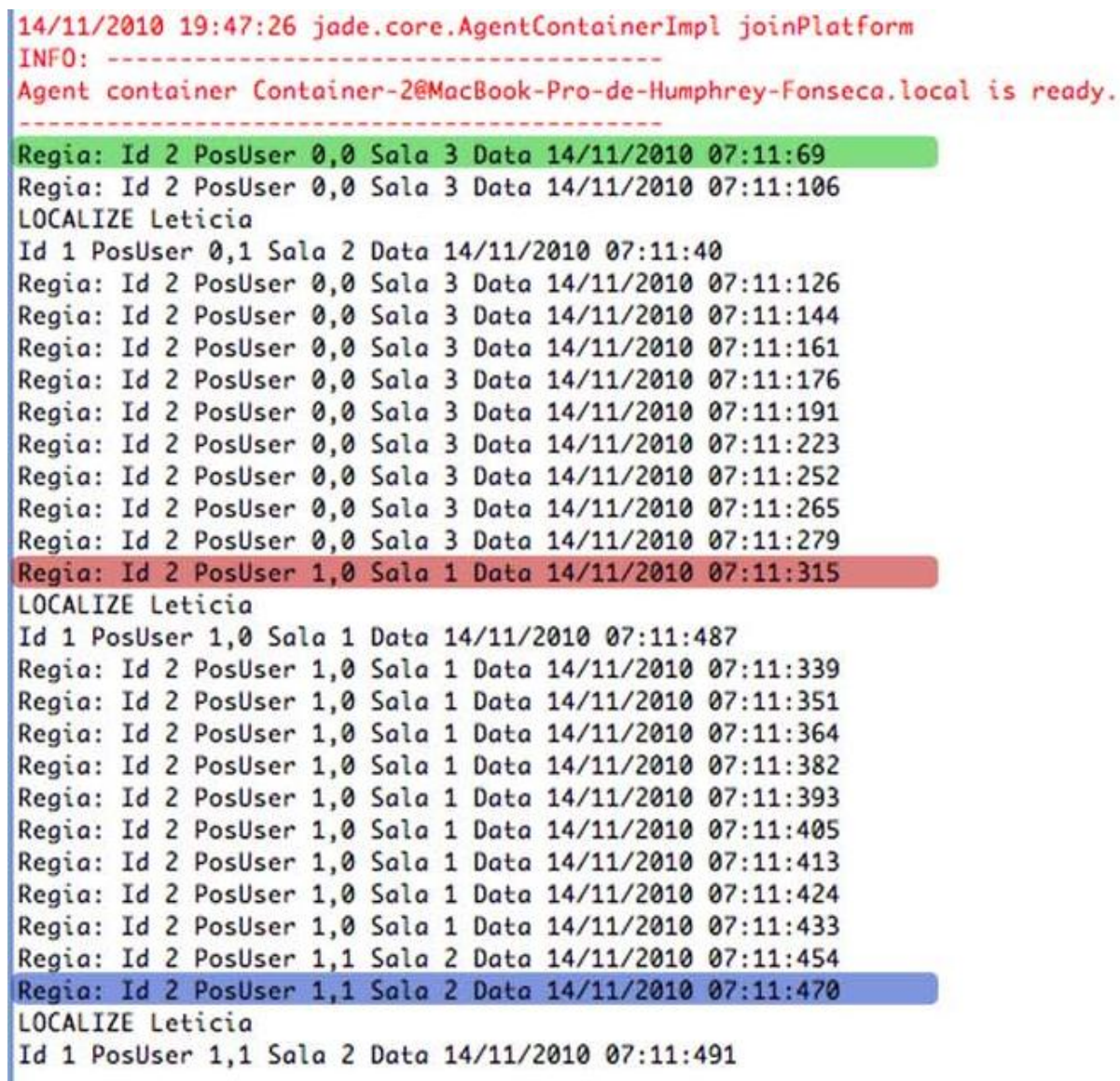

Figura 4. Screenshot do console da usuária Régia

A usuária Letícia também precisa encontrar a usuária Régia para mostrar os artigos correlatos ao trabalho que estão fazendo e solicita ao agente localizador que a encontre. A Figura 5 ilustra um screenshot do console da usuária Letícia com o monitoramento da localização da usuária Régia, a qual se movimenta pelas três salas do ambiente. 


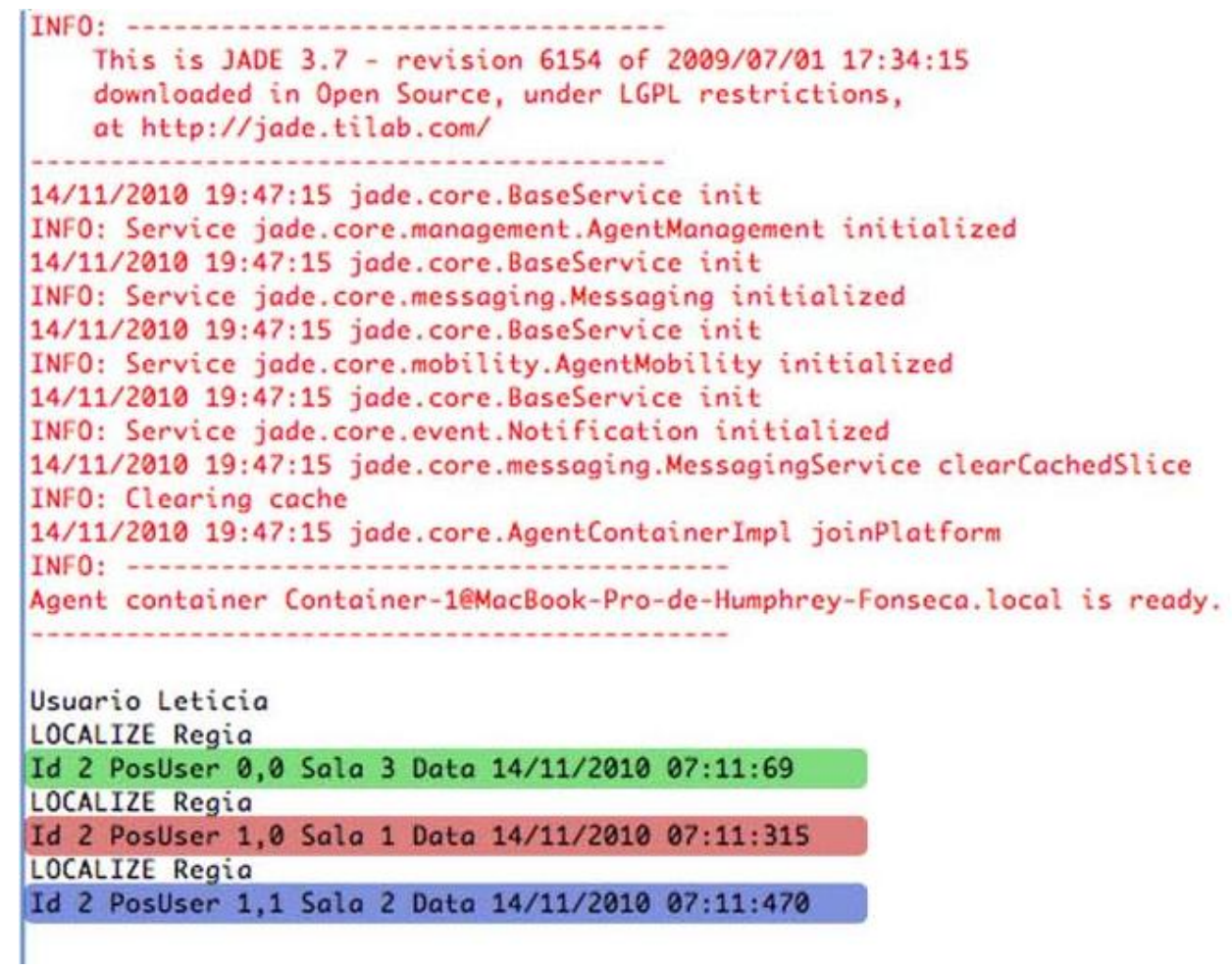

Figura 5. Screenshot do console da usuária Letícia

Note que os três elementos contextuais definidos na Seção 5 estão presentes nas Figuras 4 e 5: identificação (Id), localização (Sala) e tempo (data e hora).

\section{Conclusões e Trabalhos Futuros}

Este artigo apresentou uma simulação para a oferta de serviço de localização de usuários em um ambiente interno com uso da abordagem de SMA. Para isso, uma nova arquitetura foi definida e um protótipo desenvolvido com o uso da plataforma JADE; além da especificação de três elementos contextuais: identificação, localização e tempo, os quais auxiliaram o controle da identificação, da mobilidade e das informações sobre a orientação do usuário em um ambiente interno do prédio SG 11 da UnB.

O protótipo desenvolvido utiliza abordagem SMA, no qual, três agentes interagem para facilitar a localização do usuário: agente usuário, agente ambiente e agente localizador. $\mathrm{O}$ uso do paradigma SMA e da plataforma JADE facilitaram a realização deste projeto, devido a abstração fornecida para o desenvolvimento do serviço de localização e a simplificação que a plataforma oferece para a implementação dos agentes. Além disso, a inserção do agente reativo com estado contribuiu para a manutenção de um log com as recentes localizações dos usuários, o que permite ao usuário buscar a localização de outro no ambiente interno.

Este artigo faz parte de um projeto de pesquisa maior que tem como objetivo o uso de elementos contextuais primários e secundários para localização, identificação e oferta de serviços a usuários através de aplicações sensíveis ao contexto com uso de SMA desenvolvida dentro do laboratório ANDES (Algorithms, Networks, Distributed and Embedded Systems) do Departamento de Ciência da Computação, da Universidade 
de Brasília.

Algumas melhorias estão sendo trabalhadas para aumentar o desempenho do protótipo de localização de usuários em ambientes internos, como o uso de Redes Neurais, para obter maior exatidão na localização do usuário. No entanto, poderão ser utilizados modelos adicionais ao raciocínio dos agentes. Agentes cognitivos para alcançar uma melhor autonomia e proatividade dos agentes no sistema e o uso de ontologias para a contextualização do ambiente e oferta serviços, de acordo com os grupos de acesso e diferentes contextos em ambientes diversificados.

\section{Referências}

Abowd, G. D. e Mynatt, E. D. (2000) "Charting past, present, and future research in ubiquitous computing", ACM Transactions on Computer-Human Interaction, vol. 7, no. 1, pp. 29-58.

Bellifemine, F. L, Claire, G., e Greenwood, D., (2007) Developing multi-agent systems with JADE, John Wiley \& Sons, $1^{\mathrm{a}}$ edição.

Bulcão Neto, R. F. (2006) "Um processo de software e um modelo ontológico para apoio ao desenvolvimento de aplicações sensíveis ao contexto", Tese de Doutorado, Instituto de Ciências Matemáticas e de Computação - ICMC-USP.

Coutaz, J., Crowley, J., Dobson, S., e Garlan, D. (2005) "The disappearing computer: Context is Key", Communications of the ACM, vol. 48, no. 3, pp. 49-53.

Dey, A. K. e Abowd, G. D. (1999) "Towards a Better Understanding of Context and Context- Awareness", In HUC '99: Proceedings of the $1^{\text {st }}$ international symposium on Handheld and Ubiquitous Computing, pp. 304-307, Springer-Verlag.

Dey, A. K. (2001) "Understanding and Using Context", In Personal and Ubiquitous Computing, vol. 5, no. 1, pp. 4-7.

Faisal A, Zain Al-Abdeen Al-Fakhry, Eslam Al Maghayreh, Shadi Aljawarneh, Ahmad T. Al-Taani (2010) "A Multi-Agent Based System for Securing University Campus", In First International Conference on Intelligent Systems, Modelling and Simulation (ISMS 2010), Liverpool, Inglaterra, Jan 27-29.

Greenberg, S. (2001) "Context as a Dynamic Construct", In Human Computer Interaction, vol. 16, no. 2-4, p. 257-268.

Jih, W., Huang, C. e Hsu, J. Y. (2009) "Context Life Cycle Management in Smart Space Environments", In 3rd Workshop on Agent-Oriented Software Engineering Challenges for Ubiquitous and Pervasive Computing (AUPC'09), Londres, Inglaterra, Jul 13-17.

Loureiro, A. A. F., Oliveira, R. A. R., Silva, R. M. B., Pires Júnior, W. R., Oliveira, L. B. R. de, Moreira, R. A., Siqueira, R. G., Rocha, P. S e Ruiz, L. B. (2009) "Computação Ubíqua Ciente de Contexto: Desafios e Tendências", $27^{\circ}$ Simpósio Brasileiro de Redes de Computadores e Sistemas Distribuídos (SBRC'09), Recife PE, Mai 25-29.

Neves, A. R. M., Maia, L. T, Ralha, C. G e Jacobi, R. P. (2010) "Prototype for Indoor Localization Based on MultiAgent System", In 3rd International Conference on Intelligent and Advanced Systems (ICIAS 2010), pp. 1-4, Kuala Lumpur, Malásia, 
IEEE Computer Society, Jun 15-17.

Nguyen, T., Loke, S. W., Torabi, T. e Lu, H. (2008) "Multiagent place-based virtual communities for pervasive computing", In Sixth Annual IEEE International Conference on Pervasive Computing and Communications (PerCom'08), pp. 602608 , Hong Kong, IEEE Computer Society.

Nguyen, T., Loke, S. W., Torabi, T. e Lu, H. (2009) "PlaceAware: A Tool for Enhancing Social Interactions in Urban Places", In Proceedings of the 10th International Symposium on Pervasive Systems, Algorithms, and Networks (ISPAN'09), Kaohsiung, Taiwan, R.O.C.

Robin S., Gregory O’H., Conor M., Donnacha P. e Rem C. (2005) “ACCESS: An Agent based Architecture for the Rapid Prototyping of Location Aware Services", In Lecture Notes in Computer Science, vol. 3516/2005, pp. 695-702, Springer-Verlag.

Russel, S. J., Norvig, P. (2009), Artificial Intelligence: A Modern Approach, Pretince Hall, $3^{\text {rd }}$ edition.

Stone, P. e Veloso, M. (2000) "Multiagent Systems: A Survey from a Machine Learning Perspective", In Autonomous Robotics, vol. 8, no. 3, pp. 345-383.

Sycara, K. S. (1998) "Multiagent System", In American Association for Artificial Intelligence.

Truong, K. N., Abowd, G. D, e Brotherton, J. A. (2001) "Who, what, when, where, how: Design issues of capture \& access applications", In Proceedings of the Internacional Conference on Ubiquitous Computing (Ubicomp'01), In Lecture Notes in Computer Science, pp. 209-224, Atlanta, Georgia, EUA, Springer-Verlag.

Vladoiu, M. e Constantinescu, Z. (2010) "Learning with a Context-Aware Multiagent System", In $9^{\text {th }}$ Roedunet International Conference (RoEdNut'10), pp. $368-373$, Sibiu, Romania, Jun 24-26.

Vieira, V., Tedesco, P. e Salgado A. C. (2009) "Modelos e Processos para o Desenvolvimento de Sistemas Sensíveis ao Contexto", Jornadas de Atualização em informática (JAI'09), Porto Alegre, SBC, cap. 8.

Weyns, D., Omicini, A., Odell, J. (2007) "Environment as a first class abstraction in multiagent systems", In Autonomous Agents Multi-Agent Systems, vol. 14, no. 5, pp. 3.

Wooldridge, M. (2002), An introduction to multiagent system, John Wiley \& Sons, $2^{\text {nd }}$ edition. 